\title{
J.M. de Zafra y los orígenes de los puentes de vigas trianguladas de hormigón armado en España
}

\section{J.M. de Zafra and the origins of reinforced concrete truss bridges in Spain}

\author{
A. Burgos Núñez ${ }^{(*)}$
}

\section{RESUMEN}

Los puentes de tramos rectos formados por vigas trianguladas de hormigón armado fueron una tipología bastante utilizada en España durante el primer tercio del siglo XX. A su difusión contribuyó especialmente el prestigioso ingeniero de caminos Juan Manuel de Zafra, uno de los pioneros del hormigón armado en España.

Con motivo de la construcción de una línea de ferrocarril en Málaga, J.M. de Zafra creó una fórmula original constituida por vigas trianguladas de hormigón armado, la cual tuvo su primera aplicación en el puente sobre el río Vélez. Levantado en 1907, este inmediatamente se configuró como arquetipo y referencia fundamental para las realizaciones posteriores.

En este artículo se analizan los planteamientos estructurales y constructivos de este puente, que, todavía en pie, constituye una de las obras más significativas de la Ingeniería Civil española del siglo XX.

Palabras clave: J.M. de Zafra; hormigón armado; vigas trianguladas; puentes de tramos rectos; Diseño Estructural, Ingeniería Civil.

\section{ABSTRACT}

Reinforced concrete truss bridges were frequently built in Spain during the first third of the 2oth century. Its importance was largely due to the fact its creator and main advocate, the Spanish civil engineer Juan Manuel de Zafra, was highly regarded as one of the most important reinforced concrete designers.

In 1907, J.M. de Zafra created an original design using triangular reinforced concrete girders. It was used for the first time in the bridge over the Vélez river (belonging to a railway in Málaga, in the south of Spain). This bridge immediately rose itself as the archetype of the typology.

This paper focuses on the structural and constructive criteria applied in this bridge, which still remains as one of the most significant works of Spanish Civil Engineering of the 2oth century.

Keywords: J.M. de Zafra; reinforced concrete; trusses; straight span bridges; Structural Design; Civil Engineering.

${ }^{(*)}$ Ingeniero de Caminos e Historiador. Profesor asociado. ETS Ingeniería de Edificación. Universidad de Granada (España). Persona de contacto/Corresponding author: abn@ugr.es (A. Burgos-Núñez).

ORCID: http://orcid.org/oooo-0oo1-9236-487X (A. Burgos-Núñez).

Cómo citar este artículo/Citation: A. Burgos-Núñez (2019). J.M. de Zafra y los orígenes de los puentes de vigas trianguladas de hormigón armado en España. Informes de la Construcción, 71(553): e283. https://doi.org/10.3989/ic.70538

Copyright: ( ) 2019 CSIC. Este es un artículo de acceso abierto distribuido bajo los términos de la licencia de uso y distribución Creative Commons Reconocimiento 4.0 Internacional (CC BY 4.0). 


\section{INTRODUCCIÓN}

A comienzos del siglo XX la construcción con hormigón armado se encontraba en proceso de regularización. Técnicos de diversa procedencia trabajaban para superar el empirismo de la etapa inicial, proporcionando los principios racionales para su consolidación como técnica constructiva. Al tiempo, se ensayaban diferentes fórmulas para aplicarlo en las construcciones, en busca de su implementación más idónea.

En el ámbito de los puentes, su uso se estaba extendiendo rápidamente para realizaciones de pequeñas dimensiones, utilizando siempre modelos de tramos rectos isostáticos con una sección transversal (casi obligada) en $\pi$. Para luces mayores se habilitaron soluciones en arco, aunque en estos casos el uso del nuevo material introducía no poca incertidumbre. En su diseño no se contemplaban adecuadamente importantes propiedades como su monolitismo, derivándose de ello una indefinición de su comportamiento (1).

En buena parte por ello, se buscaron alternativas a los puentes en arco para luces importantes. Algunas se inspiraron en el ámbito de la construcción metálica. Los tramos rectos de vigas trianguladas eran por entonces solución prácticamente universal, aplicada en todo tipo de puentes. Con cierta lógica, se hicieron propuestas para su implementación con hormigón armado. En el agitado mundo empresarial de los comienzos del nuevo material hubo incluso quienes (sobre todo en el ámbito centroeuropeo) lograron materializar con éxito modelos de construcción industrializada (2).

En España los puentes de vigas trianguladas de hormigón armado tuvieron mayor aceptación que en otros países. En gran parte, esto se debió al impulso que dio a la tipología uno de los más grandes ingenieros nacionales, Juan Manuel de Zafra.
Este insólito desarrollo tuvo como punto de partida el puente sobre el río Vélez que J.M. de Zafra proyectó en 1907 para los Ferrocarriles Suburbanos de Málaga (figura 1).

Esta obra singular se constituyó como arquetipo y sirvió de base para la construcción de otros puentes similares, hasta que en 1927 se estandarizara su diseño en una colección de modelos oficiales. A ella se ajustarían un importante número de puentes construidos en los años 1930.

\section{J. M. DE ZAFRA, UNA FIGURA CLAVE DE LA INGENIERÍA CIVIL ESPAÑOLA}

Juan Manuel de Zafra y Esteban (Huelva, 1869-Madrid, 1923) es una de las grandes personalidades históricas de la Ingeniería Civil española (figura 2).

Su figura es importante desde una triple dimensión. Por un lado, en su faceta como ingeniero en ejercicio, entre 1892 y 1908 desplegó una notable actividad. Compaginó su trabajo en el Puerto de Sevilla con una destacada trayectoria como consultor especializado en construcciones de hormigón armado. Su lista de realizaciones, no muy amplia pero sí trascendentalmente significativa, se concretó en la línea de Ferrocarril de las Minas de Cala y en la de los Ferrocarriles Suburbanos de Málaga, que incorporaban puentes, depósitos y varios embarcaderos. Todos ellos fueron resueltos airosamente, y siempre desde la más rigurosa racionalidad, con el nuevo material de construcción (3).

En 1909 se incorporó al plantel de profesores de la Escuela de Caminos, donde a partir de entonces desarrolló una brillante carrera docente. Apoyada tanto en poderosos fundamentos científicos como en una fecunda experiencia en el ejercicio de la profesión, su docencia constituyó un hito, ejerciendo una

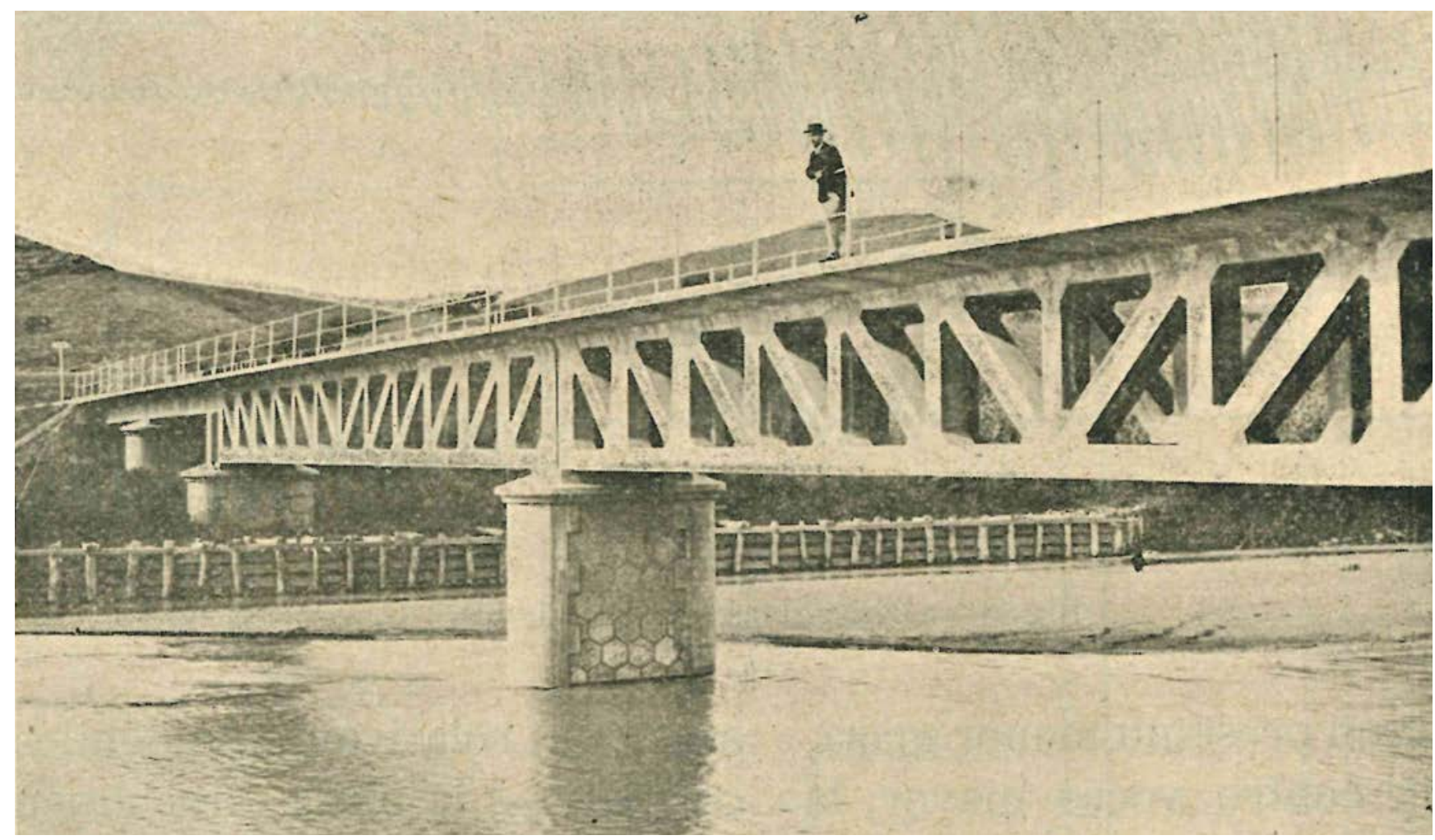

Figura 1. Puente sobre el río Vélez en 1909. Revista de Obras Públicas. Biblioteca del Hospital Real. Universidad de Granada. 


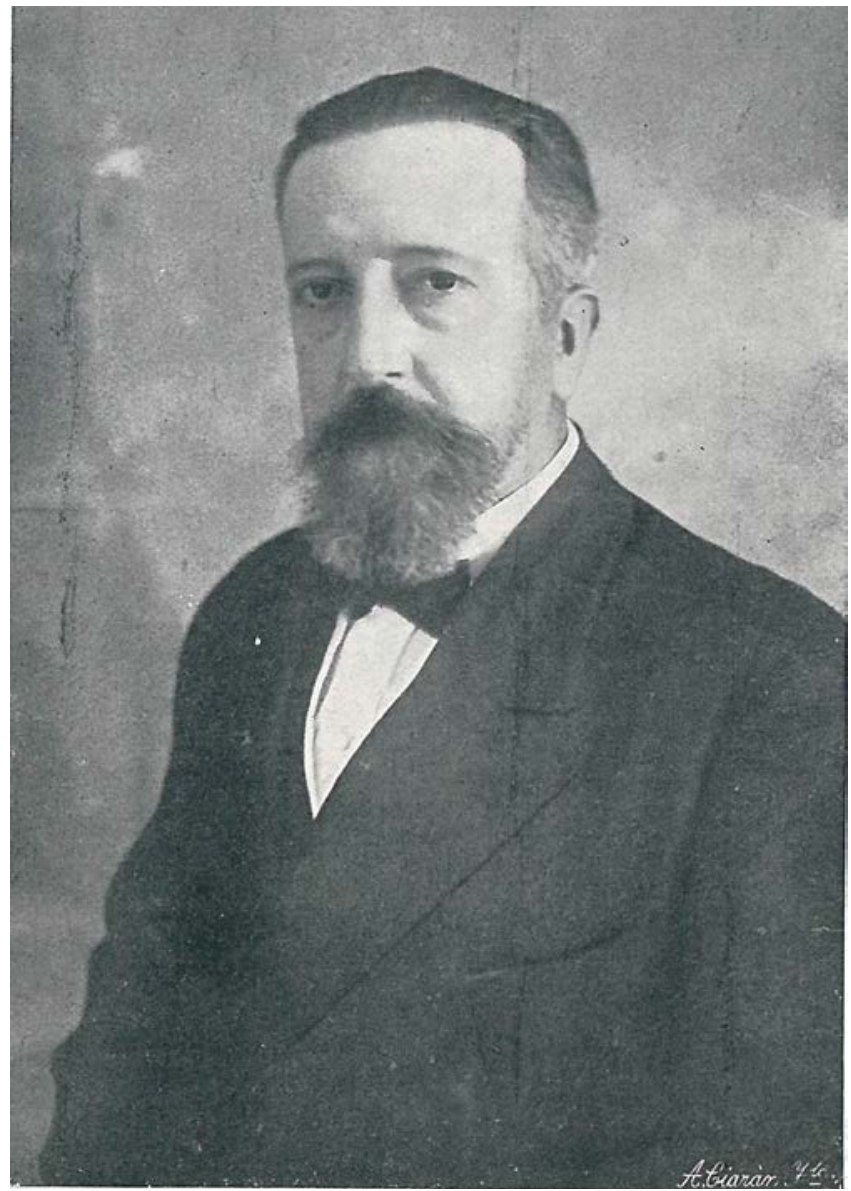

Figura 2. Juan Manuel de Zafra en 1919, fotografía realizada con ocasión de su ingreso en la Real Academia de Ciencias. Revista de Obras Públicas. Biblioteca del Colegio de ingenieros de Caminos, Canales y Puertos. Sede Central.

influencia trascendental en la formación de los grandes ingenieros civiles españoles del siglo XX (4).

Su notable contribución al progreso de la Ingeniería Civil en España fue agrandada con una valiosa y oportuna producción científica, siempre enfocada desde y para el ejercicio de la profesión. Su ingreso en la Academia de Ciencias en 1919 constituiría su reconocimiento oficial.

\section{ZAFRA Y LOS PUENTES \\ DE LOS FERROCARRILES SUBURBANOS DE MÁLAGA}

En 1905 un consorcio de inversores mayoritariamente belgas apostó por la formación de una red de ferrocarriles de vía estrecha en torno a la ciudad de Málaga (5). Inmediatamente emprendieron la construcción de la primera de sus líneas, que debía enlazar la capital provincial con la localidad costera de Torre del Mar. Se trataba de una obra muy condicionada por la circunstancia de que los $35 \mathrm{~km}$ de su trazado tendrían que discurrir prácticamente en su totalidad literalmente por playas.

En el diseño de sus puentes esto fue, efectivamente, muy determinante, ya que las numerosas ramblas que interceptaban el trayecto debían ser atravesadas próximas a su desembocadura, en una zona de pendiente transversal prácticamente nula. La única solución que garantizaba una importante sec- ción de desagüe sin elevar la rasante era la de tramos rectos. Por otro lado, en muy pocos de los emplazamientos el terreno permitía la cimentación de los estribos de un arco. De este modo, en la carretera de Málaga a Almería, recientemente dispuesta por la misma estrecha faja costera, se había optado mayoritariamente por los tramos rectos. Parecía ser, pues, este, el único esquema estructural posible, pero su materialización con elementos metálicos se había revelado comprometida por los efectos de la corrosión marina. Esta acumulación de circunstancias llevó al ingeniero Leopoldo Werner, proyectista de la línea, a apostar por el hormigón armado, proponiendo tramos rectos de 8 y $10 \mathrm{~m}$ de luz, apoyados isostáticamente, para todos los puentes de la línea (6).

Pero Werner no estaba familiarizado con el nuevo material, por lo que presentó unos diseños muy teóricos, de ejecución problemática. Además, dejó sin definir el puente sobre el río Vélez, cuyo franqueo no podía resolverse con los sencillos tramos rectos de pequeña luz. La Compañía de los Ferrocarriles Suburbanos de Málaga recurrió entonces a J.M. de Zafra, quien por entonces ya sobresalía como gran especialista en construcciones de hormigón armado.

Zafra trabajó para los Ferrocarriles de Málaga desde 1907 hasta 1911, desempeñando un trabajo que tendría especial trascendencia para la Ingeniería de Puentes española del siglo XX. Allí concibió su modelo de tramos rectos con sección transversal en $\pi$, como optimización de los puentes esbozados por Werner. Hasta la generalización de las vigas prefabricadas de hormigón pretensado, sería el patrón más utilizado en España para la construcción de puentes económicos.

También acabaría convirtiéndose en arquetipo otra trascendental creación suya para los Ferrocarriles de Málaga: el puente sobre el río Vélez. Si bien esta tendría una menor repercusión, con ella arrancaría en nuestro país la tipología de puentes de vigas trianguladas de hormigón armado.

\section{EL PUENTE SOBRE EL RÍO VÉLEZ, DISEÑO GENERAL}

El trazado ferroviario tenía que cruzar el río Vélez cerca de la población de Torre del Mar, un centenar de metros aguas arriba de un puente metálico de carretera, proyectado en la década de 1860 y por problemas constructivos terminado casi veinte años más tarde (figura 3).

La existencia previa de este puente de tramos rectos de celosía fue bastante útil para el diseño del nuevo. Ciertos aspectos, como la sección de desagüe, se determinaron a partir de la experiencia previa con el puente metálico. Estimada por Zafra en $359 \mathrm{~m}^{2}$ la sección necesaria, dada la forma del emplazamiento se infería que el puente nuevo debía tener una luz de alrededor de cien metros (7).

En otras ramblas anchas esta distancia había podido salvarse con tramos rectos de sección $\pi$ concatenados (Arroyo Totalán, 6 vanos de $10 \mathrm{~m}$ de luz) pero en el río Vélez esto no era posible. Su cauce era muy inestable, habiendo sufrido el puente de la carretera continuas socavaciones de sus pilas.

Consciente de esta debilidad, Zafra procuró colocar el menor número posible de pilas en el cauce. Utilizó todo lo posible los tramos $\pi$ en las aproximaciones y la parte central la resolvió con dos tramos de mayor longitud, apoyados en una 


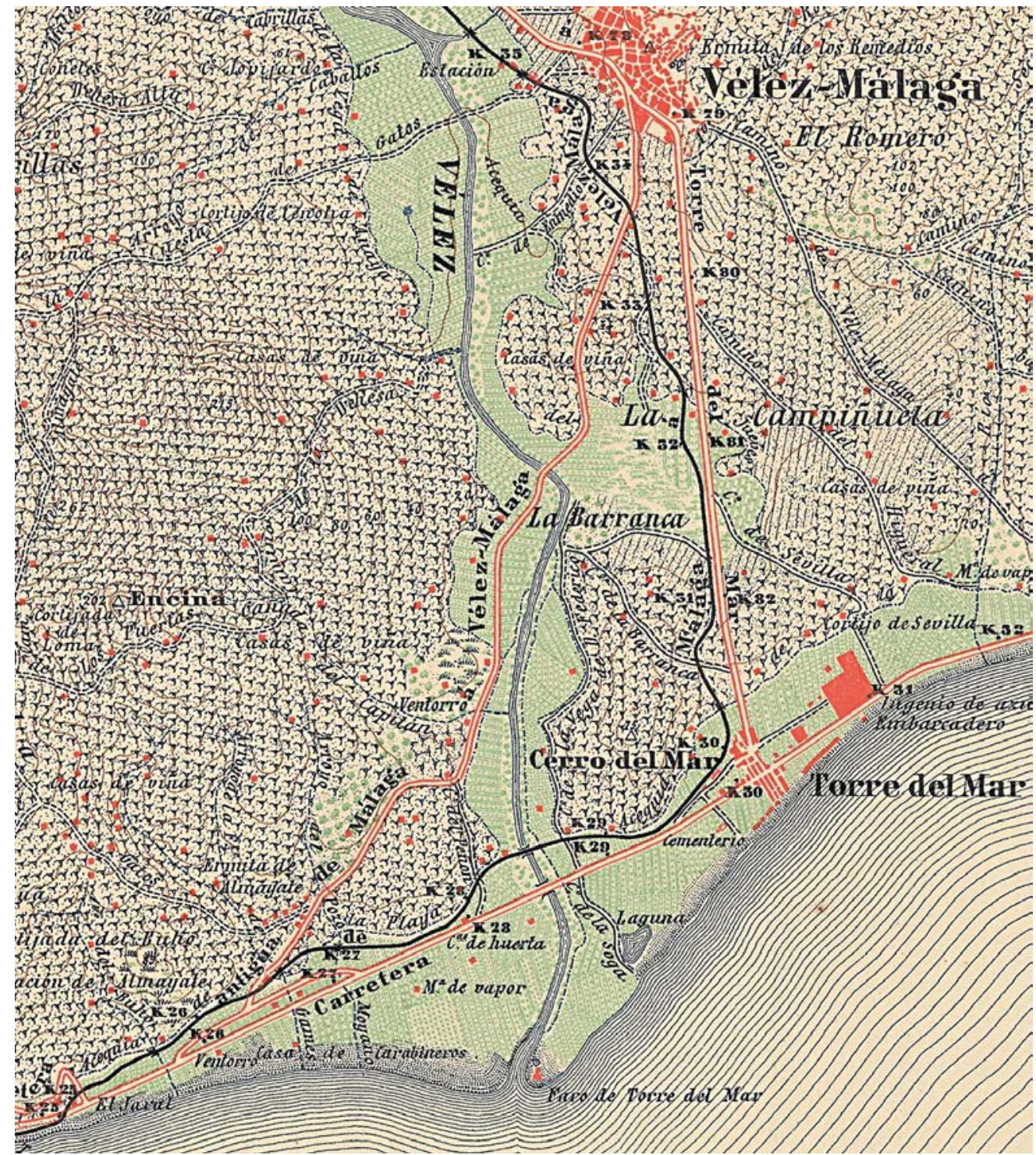

Figura 3. Infraestructuras de transporte en la desembocadura del río Vélez. Detalle de la hoja 1054 del MTN, confeccionada en 1916. Instituto Geográfico Nacional de España.

única pila (7). De este modo, el diseño final se acomodaba a la asimetría del emplazamiento con dos tramos $\pi$ en la margen derecha, dos tramos centrales de 26,50 m de luz y tres tramos $\pi$ en la margen izquierda (figura 4).

Con esta disposición quedaban resueltos todos los condicionantes de partida, pero había que habilitar una solución para los tramos de $26,50 \mathrm{~m}$, luz que en puentes de hormigón armado hasta entonces sólo se había podido alcanzar con arcos.
Descartados estos por las razones ya comentadas, Zafra apostó por una original transposición al nuevo material del más contrastado y por entonces reiteradamente aplicado esquema estructural para puentes: las vigas trianguladas metálicas.

Para ello se apoyaría en algunas realizaciones anteriores. En 1898 el ingeniero francés Harel de la Nöe había construido su famoso puente en $\mathrm{X}$ de Mans, un primitivo puente mixto formado por dos vigas metálicas de alma llena conectadas con

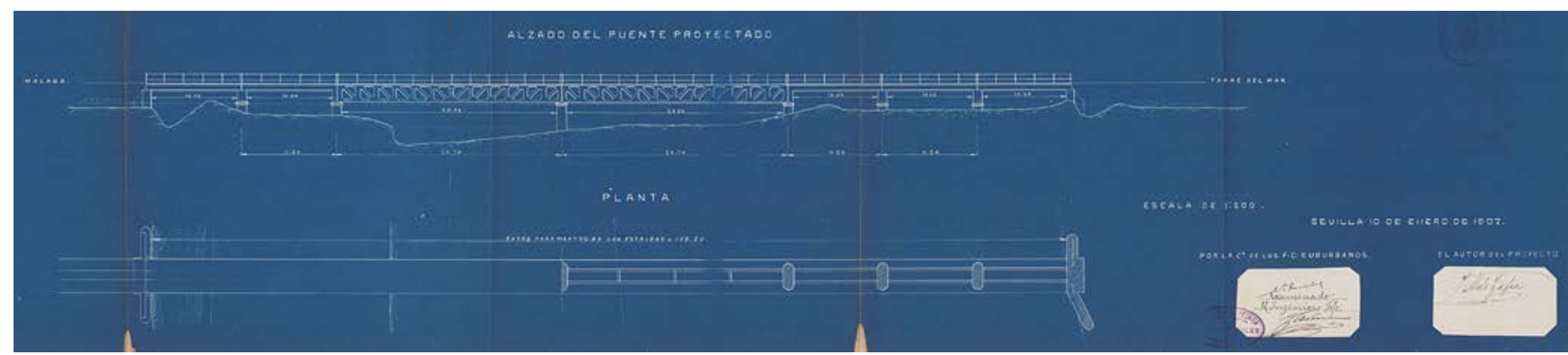

Figura 4. J.M. de Zafra, Proyecto del puente sobre el río Vélez, 1907.Alzado y planta general. Archivo General de la Administración, 04.087 .25 
una rudimentaria losa de hormigón armado a modo de tablero (8). Como el mismo Zafra reconocería, tal fue el esquema básico para su puente del río Vélez (7), pero con otro tipo de vigas como cuchillos.

Otro referente básico para Zafra serían los reforzamientos de los puentes de la línea férrea de Courcelles al Campo de Marte, París, llevados a cabo por Charles Rabut en 1900. Su propuesta, aunque en la misma línea de los remotos puentes mixtos, se implicaba con mayor intensidad en la exploración de las posibilidades de la combinación de metal y hormigón, lo que proporcionó a Zafra el argumento definitivo.

Rabut recurrió en efecto a colocar un tablero de hormigón armado sobre los cuchillos metálicos existentes, pero estos serían además reforzados mediante su anegamiento en hormigón. Se comprobó experimentalmente la validez de este procedimiento, registrando notables reducciones (en una cantidad cercana al 50\%) de las deformaciones obtenidas en puentes metálicos antes de su transformación (9).

Tales experiencias fueron, efectivamente, concluyentes para Zafra, llevándole a proponer para su puente cuchillos de metal hormigonado (o sea, vigas trianguladas metálicas envueltas en hormigón), unidos y arriostrados por sus cordones superiores mediante una losa de hormigón armado (7) (figuras 5 y 6).
Zafra no se limitó, no obstante, a reforzar tramos metálicos al uso. Las vigas del puente del río Vélez, original e intencionadamente concebidas en metal hormigonado, si bien participaban de algunos principios de diseño de las estructuras metálicas, presentaban con ellas notables diferencias, especialmente desde el punto de vista constructivo.

Los esfuerzos de diseño se determinaron mediante procedimientos de Estática Gráfica (figura 7), para un tramo isostático y con arreglo a las cargas gravitatorias (y la componente vertical del viento) prescritas en la Instrucción de puentes metálicos de 1902.

A partir de estos esfuerzos generales, y asumiendo la hipótesis de uniones articuladas (considerando así a efectos de cálculo un sistema estáticamente determinado), se dedujeron las fuerzas equivalentes actuantes sobre cada elemento. Concretamente, por ejemplo, los axiles en los cordones se obtenían dividiendo el momento flector por el brazo de palanca conformado por la altura de la viga (7).

Obtenidas así las solicitaciones equivalentes en las barras, sólo restaba dividir por la tensión de trabajo para determinar la sección transversal. Tomando en consideración las conclusiones de Rabut, Zafra asumió que gracias a la colaboración resistente del hormigón "en un puente de este tipo las secciones de los elementos metálicos pueden ser la mitad de las

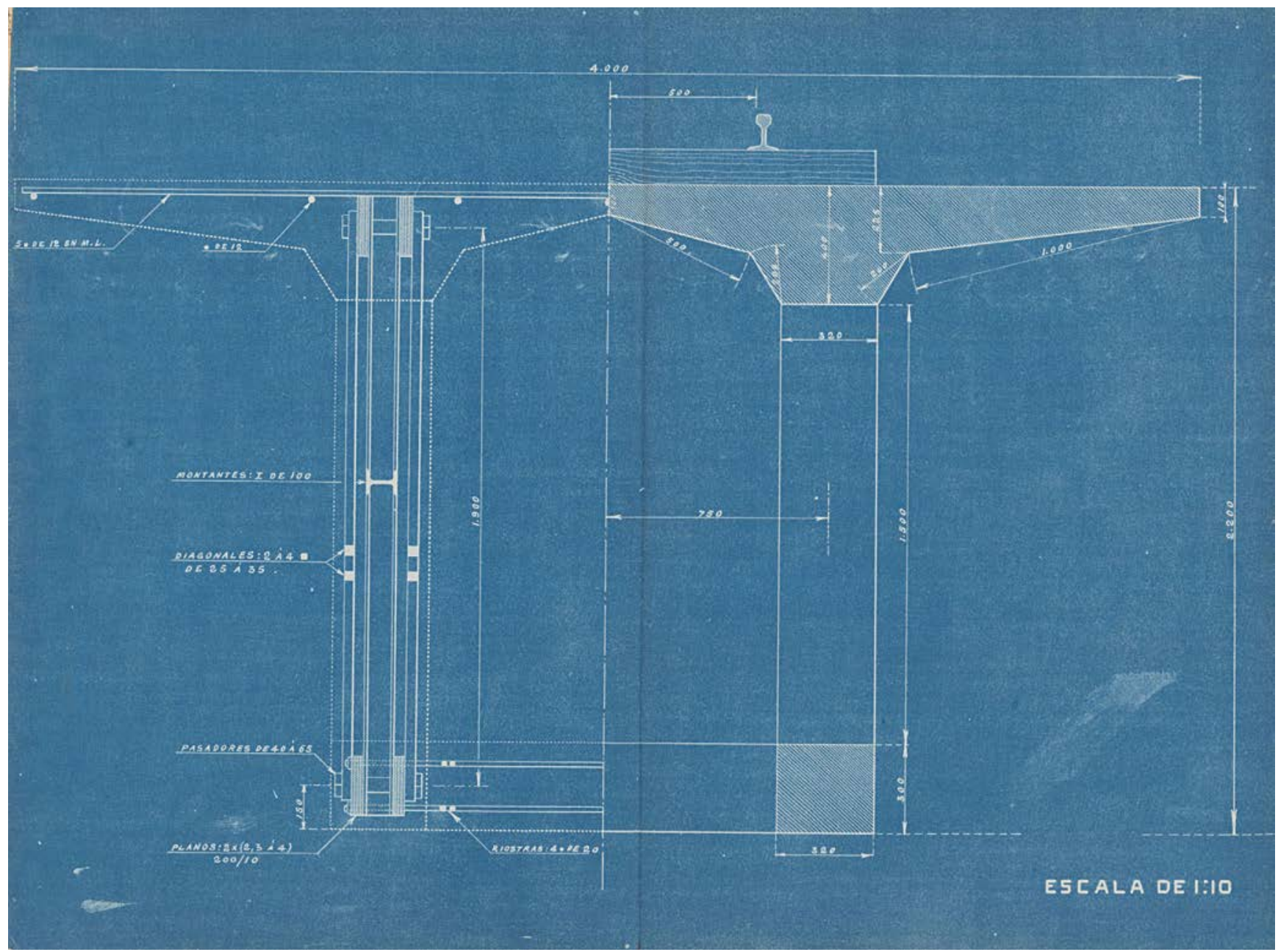

Figura 5. J.M. de Zafra, Proyecto del puente sobre el río Vélez, 1907. Sección transversal. Archivo General de la Administración, 04.087.25/8094. 


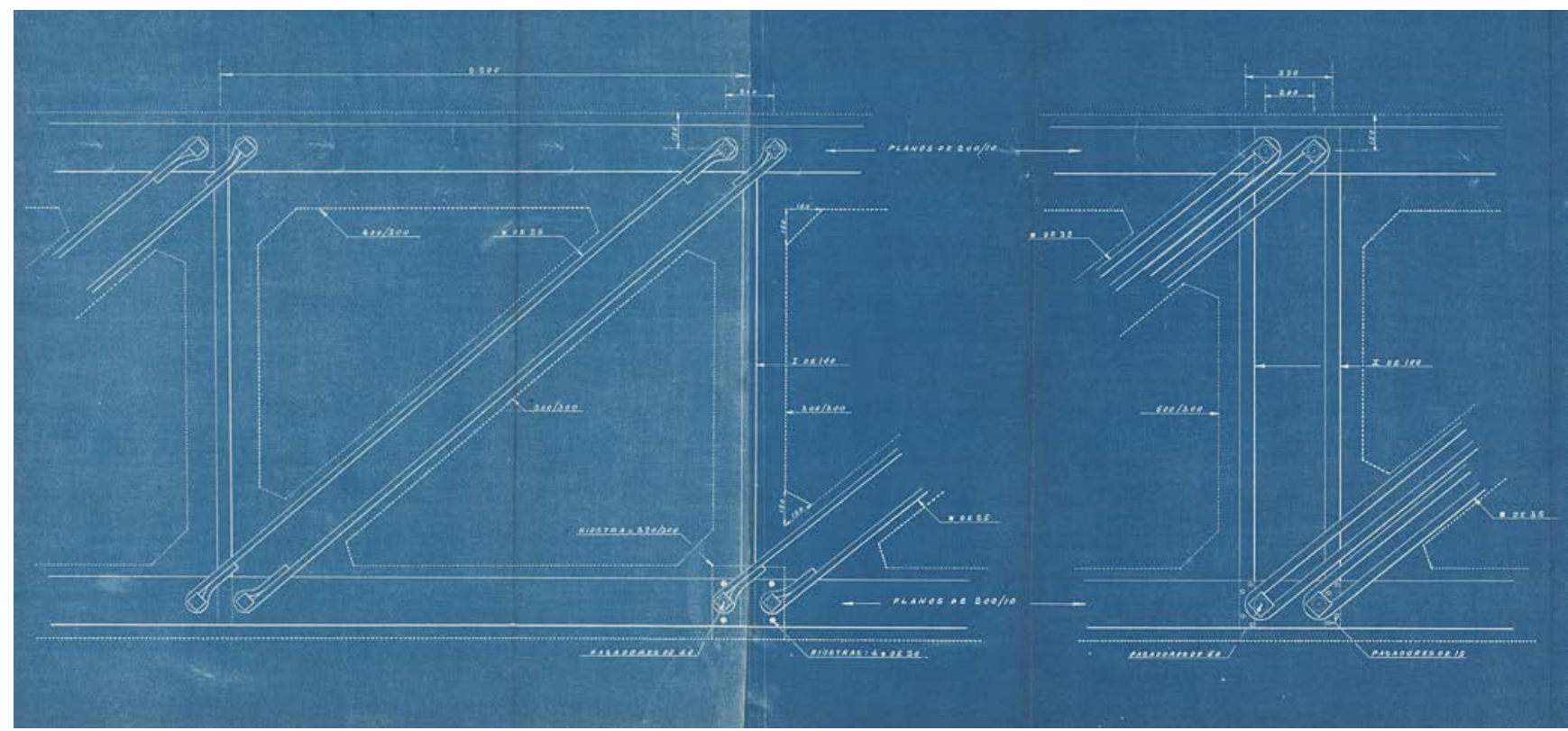

Figura 6. J.M. de Zafra, Proyecto del puente sobre el río Vélez, 1907. Constitución de las vigas trianguladas. Archivo General de la Administración, 04.087.25/8094.

correspondientes a un puente ordinario, o lo que es igual, se pueden calcular admitiendo un trabajo aparente doble del usual"(7). Por las suspicacias que entonces despertaba el hormigón no quiso llegar a tanto y adoptó para los elementos metálicos una tensión de trabajo de $125 \mathrm{MPa}$, no obstante muy por encima de los 90 MPa prescritos en la normativa entonces vigente, la Instrucción de Puentes Metálicos de 1902.

La integración monolítica de las piezas de acero en el metal hormigonado las eximía de los requisitos constructivos propios de las estructuras metálicas, siendo dimensionadas racionalmente conforme a su función estructural.

De este modo, los cordones de los cuchillos pudieron hacerse con la sección estrictamente necesaria. Se acoplaron más pletinas de chapa en el centro del vano (donde la flexión es máxima), reduciéndose paulatinamente su número en dirección a los apoyos (figura 7). Los cordones superior e inferior presentaban la misma disposición de pletinas.

De acuerdo con el reparto teórico de esfuerzos equivalentes, a los montantes y diagonales les correspondían esfuerzos axiles generados por el cortante. Por facilidad de construcción, Zafra había optado por una triangulación Pratt, en la que los montantes trabajan a compresión. Estos elementos, aunque incorporaban un perfil metálico, fueron diseñados teniendo en cuenta sólo el trabajo del hormigón. Su sección iba disminuyendo desde los montantes de los extremos a los del centro del vano. Para las diagonales, sometidas a tracción, se observó el criterio contrario y fueron resueltas con barras de acero de sección cuadrada a modo de horquillas, enganchadas mediante pasadores a las pletinas de los cordones (figuras 5 y 6 ).

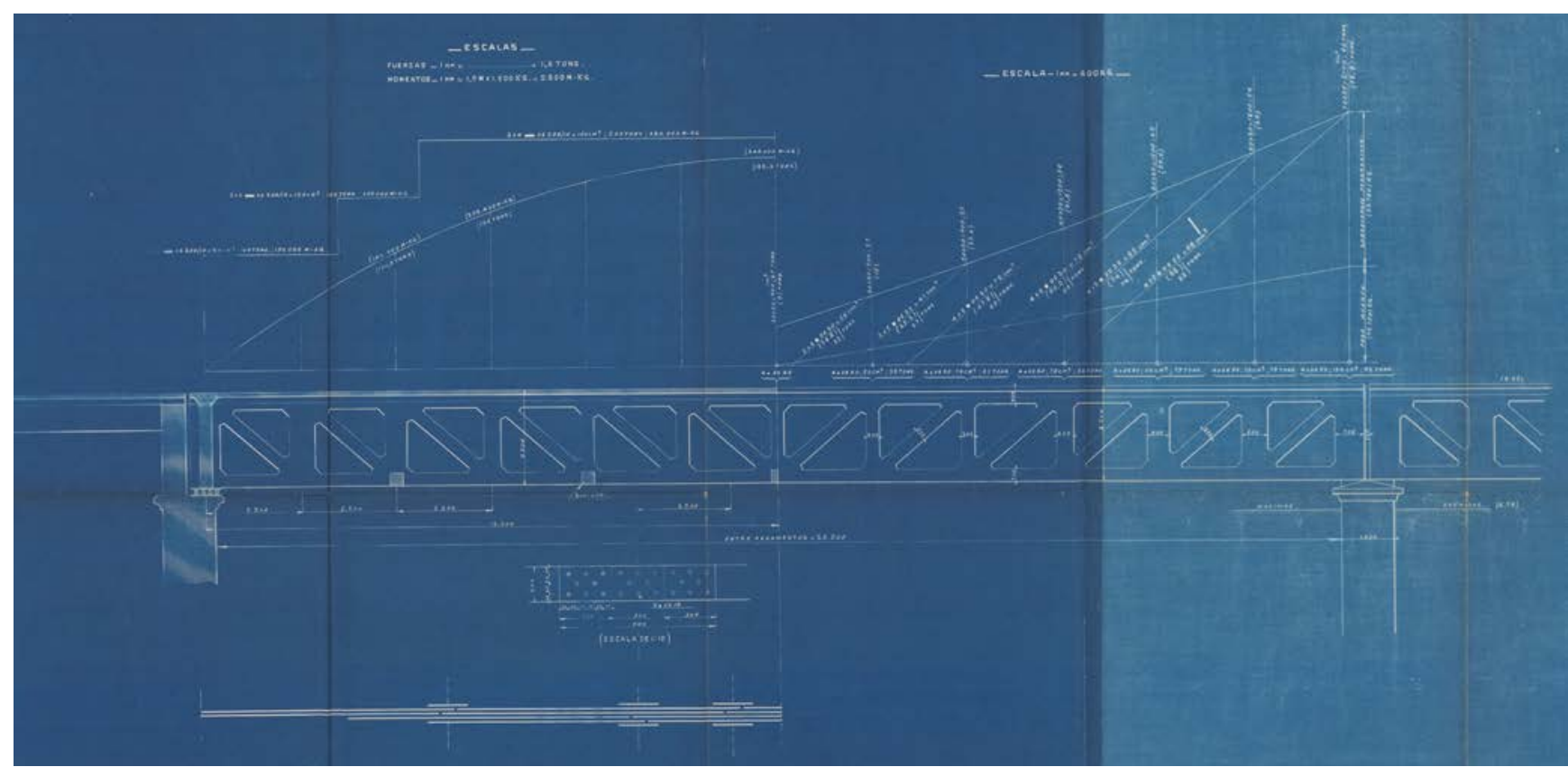

Figura 7. J.M. de Zafra, Proyecto del puente sobre el río Vélez, 1907. Representación gráfica de las leyes de esfuerzos flectores y cortantes en los tramos de 26,50 m. Archivo General de la Administración, 04.087.25/8094 
A juicio de Zafra, las simplificaciones descritas no restaban validez al procedimiento general de cálculo. Posteriormente, en uno de sus clásicos tratados, lo definiría como completamente racional y satisfactorio, así como conducente al aprovechamiento idóneo tanto de hormigón como de acero (10).

La estructura del puente se completaba, como en los puentes mixtos, con un tablero de losa de hormigón armado, firmemente unida a los cuchillos (figura 6). El procedimiento debería garantizar la resistencia ante acciones horizontales, a lo que también contribuirían varias riostras alternadas entre los cordones inferiores y unos tabiques transversales de cierre en los extremos de las vigas (figura 8).

Basándose en su gran intuición estructural (y en el buen comportamiento de otras obras suyas, como el embarcadero de las Minas de Cala) confiaba en que, al quedar todos sus elementos envueltos en hormigón, se conseguía su colaboración mutua y la respuesta solidaria ante los esfuerzos secundarios, inestabilidad o flexiones transversales. De este modo, en la práctica todas esas solicitaciones locales quedaban suprimidas.

\section{MATERIALIZACIÓN INICIAL Y APLICACIONES POSTERIORES}

La construcción del puente se verificó durante el año 1907, quedando la línea inaugurada en enero de 1908 (figura 9).

Los reconocimientos practicados con ocasión de esta investigación revelan que la materialización de la obra se ajustó escrupulosamente al diseño concebido por Zafra.

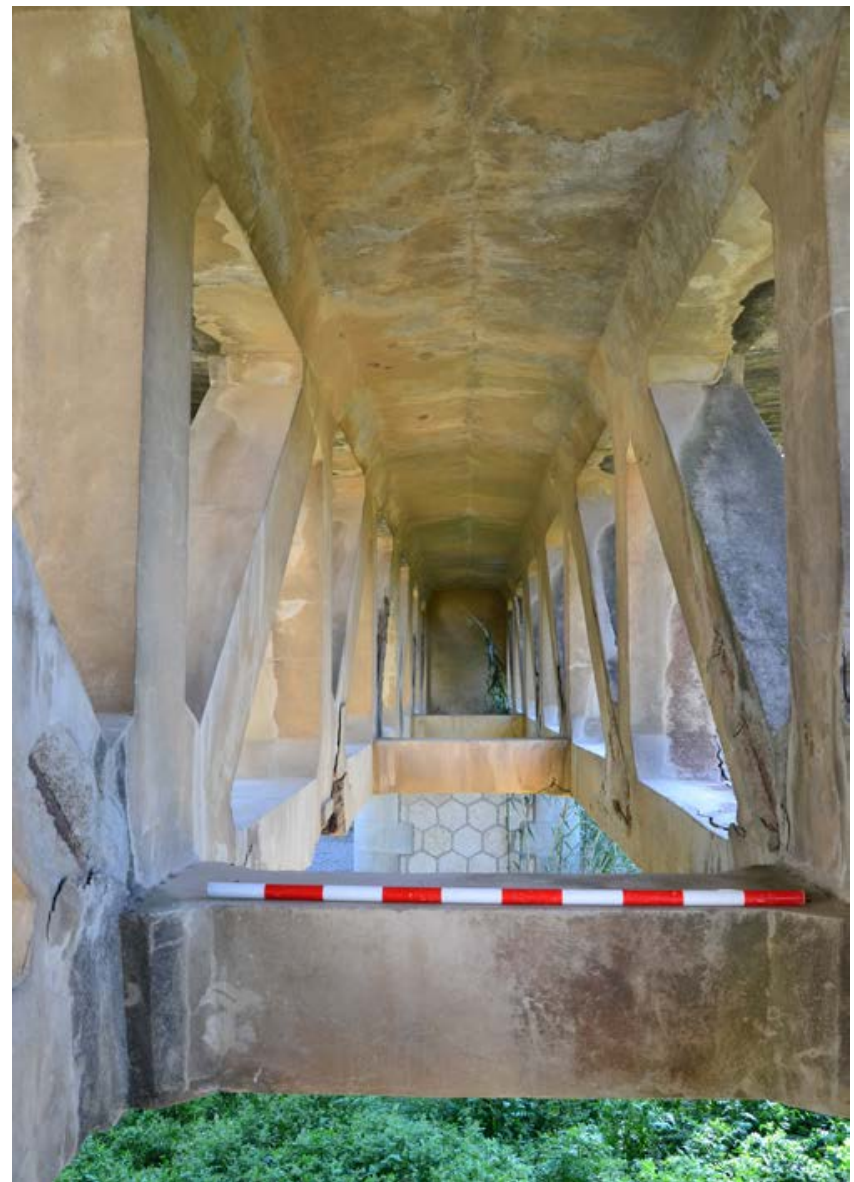

Figura 8. Riostras inferiores de unión de los cuchillos. Fotografía del autor.

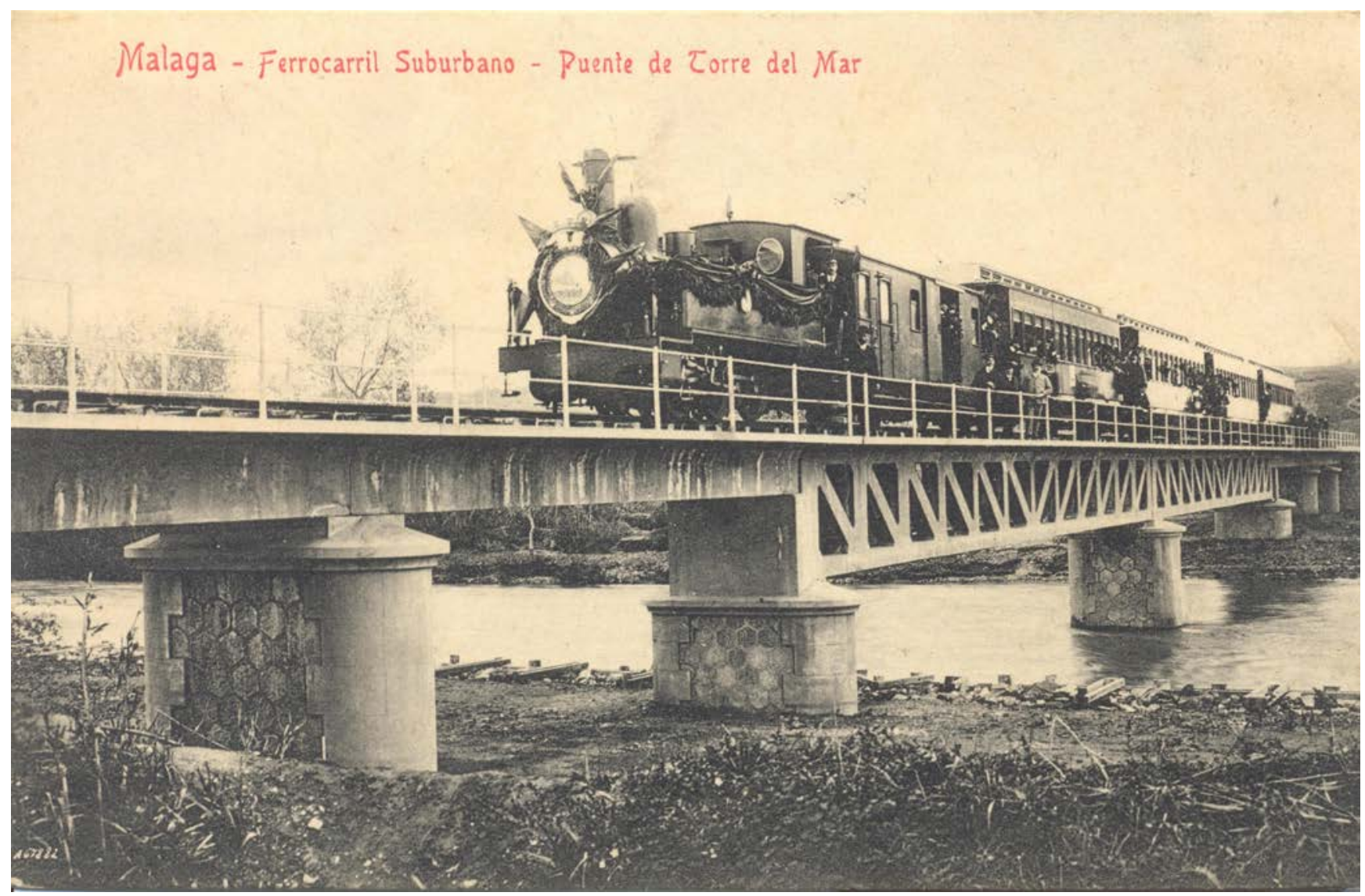

Figura 9. Puente sobre el río Vélez en su inauguración. Postal comercial, colección del autor. 


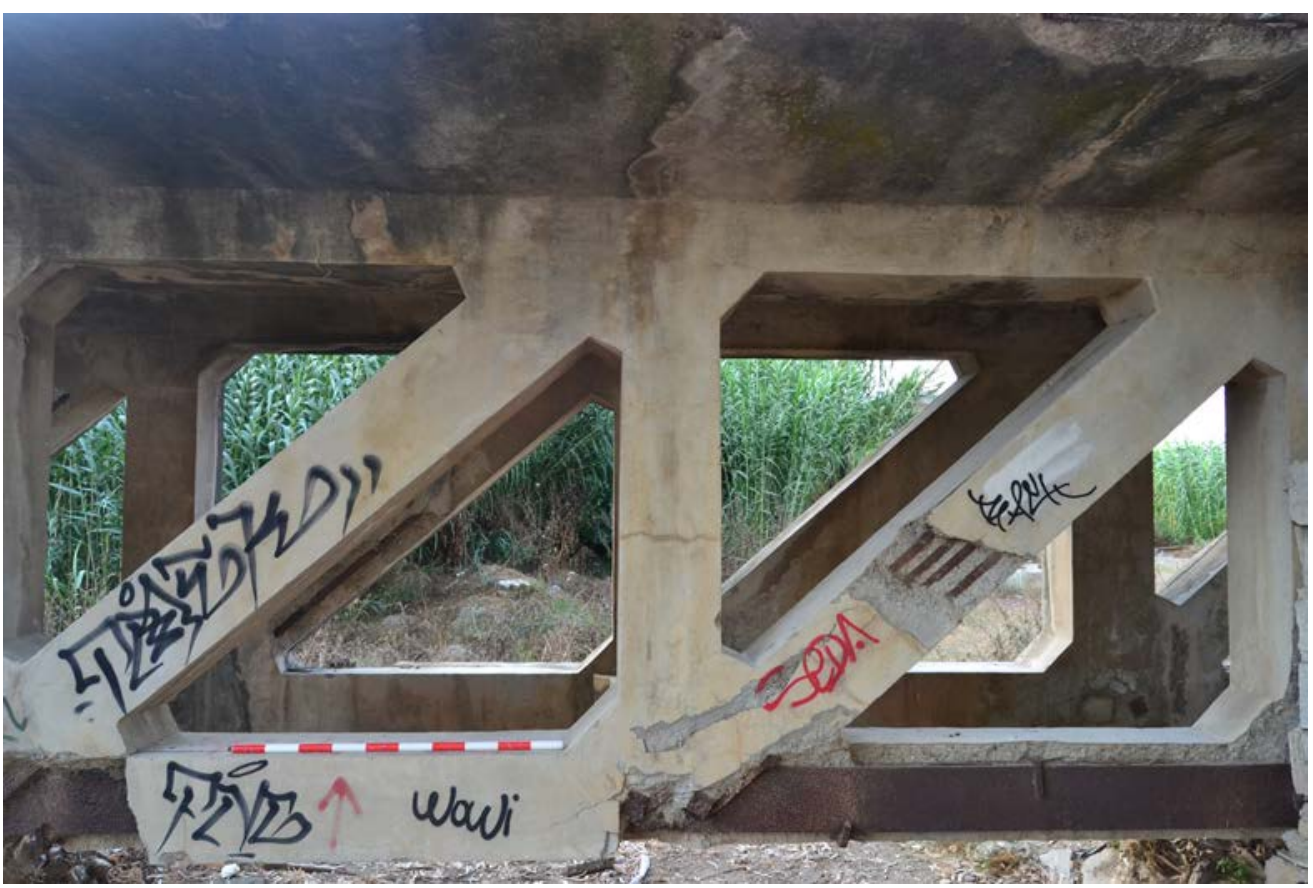

Figura 10. Elementos de la estructura del puente, visibles por los desperfectos. Fotografía del autor.

El avanzado estado de deterioro en que se encuentra el puente en la actualidad ha dejado al descubierto gran parte de sus elementos metálicos, habiéndose comprobado que su disposición, forma y dimensiones son exactamente las prescritas en el proyecto (figura 10). Otra muestra más de la solvencia y profesionalidad de J.M. de Zafra, esta vez como ingeniero de obra.

La afortunada resolución del paso del río Vélez consagró las vigas trianguladas como fórmula canónica, siendo utilizada desde entonces (junto con el otro modelo de sección $\pi$ ) en los puentes de las nuevas líneas de la compañía.

De este modo, en 1909 el propio Zafra dirigió la construcción de sendos viaductos en los dos brazos de la desembocadura del Guadalhorce, utilizando en ellos tres y cuatro vigas trianguladas idénticas a las del río Vélez, respectivamente (11).

Su sucesor al frente de la gestión técnica de la compañía, el ingeniero militar Francisco Echecopar los utilizó en cinco puentes más, todos en la provincia de Málaga. Aunque los condicionantes no eran ya los mismos (todos se encontraban en el interior, alejados del mar y en emplazamientos de laderas con pendiente) se siguió implementando al milímetro la fórmula de Zafra.

\section{CONCLUSIONES SOBRE EL COMPORTAMIENTO ESTRUCTURAL Y TRASCENDENCIA DEL MODELO}

De los ocho puentes construidos con estas vigas trianguladas, siete cumplieron satisfactoriamente su función hasta el cierre de las líneas, que tuvo lugar en 1968. En uno de ellos, no obstante, el viaducto del Arroyo Hondo, las vigas trianguladas tuvieron que ser sustituidas por arcos en 1942(12).

Debieron aquí adelantarse por algún motivo los graves problemas de durabilidad que hoy, después de medio siglo sin mantenimiento, se muestran con terrible gravedad en todos los puentes que han sobrevivido.

Como ya señalaron algunos especialistas en su momento, el hecho de considerar a efectos de cálculo el puente como una estructura con nudos articulados no era del todo exacto, existiendo esfuerzos secundarios que provocaban la aparición de grietas incontroladas y el subsiguiente deterioro de las armaduras (13) (figuras 10 y 11).

Aunque se trata de una cuestión a dilucidar mediante procedimientos analíticos (cosa prevista para una segunda fase de esta investigación), no es descabellado manifestar, dado el satisfactorio comportamiento que mostraron a lo largo de su vida útil, que estructuralmente la fórmula era viable y que con las oportunas labores de mantenimiento se habría garantizado su perduración.

\section{TRASCENDENCIA DEL DISEÑO DE J.M. DE ZAFRA}

La escasez de acero provocada por la Primera Guerra Mundial favoreció la implantación de este tipo de puentes en la segunda década del siglo XX. Siempre con el modelo creado por Zafra como referencia, se construyeron diversos ejemplares repartidos por toda la Península (13).

Sin embargo, en estas obras se fueron introduciendo modificaciones lógicas, como la inevitable sustitución de los perfiles y pletinas por barras de acero. De esta forma quedaba consolidaba definitivamente el modelo como una construcción genuina de hormigón armado.

Convencido de su idoneidad, Zafra los propuso en la Colección oficial de modelos de puentes de hormigón armado para carreteras como opción idónea para tramos rectos de cierta luz.

Introdujo sin embargo notables modificaciones respecto de la fórmula primigenia puesta en práctica en el río Vélez. Aparte 


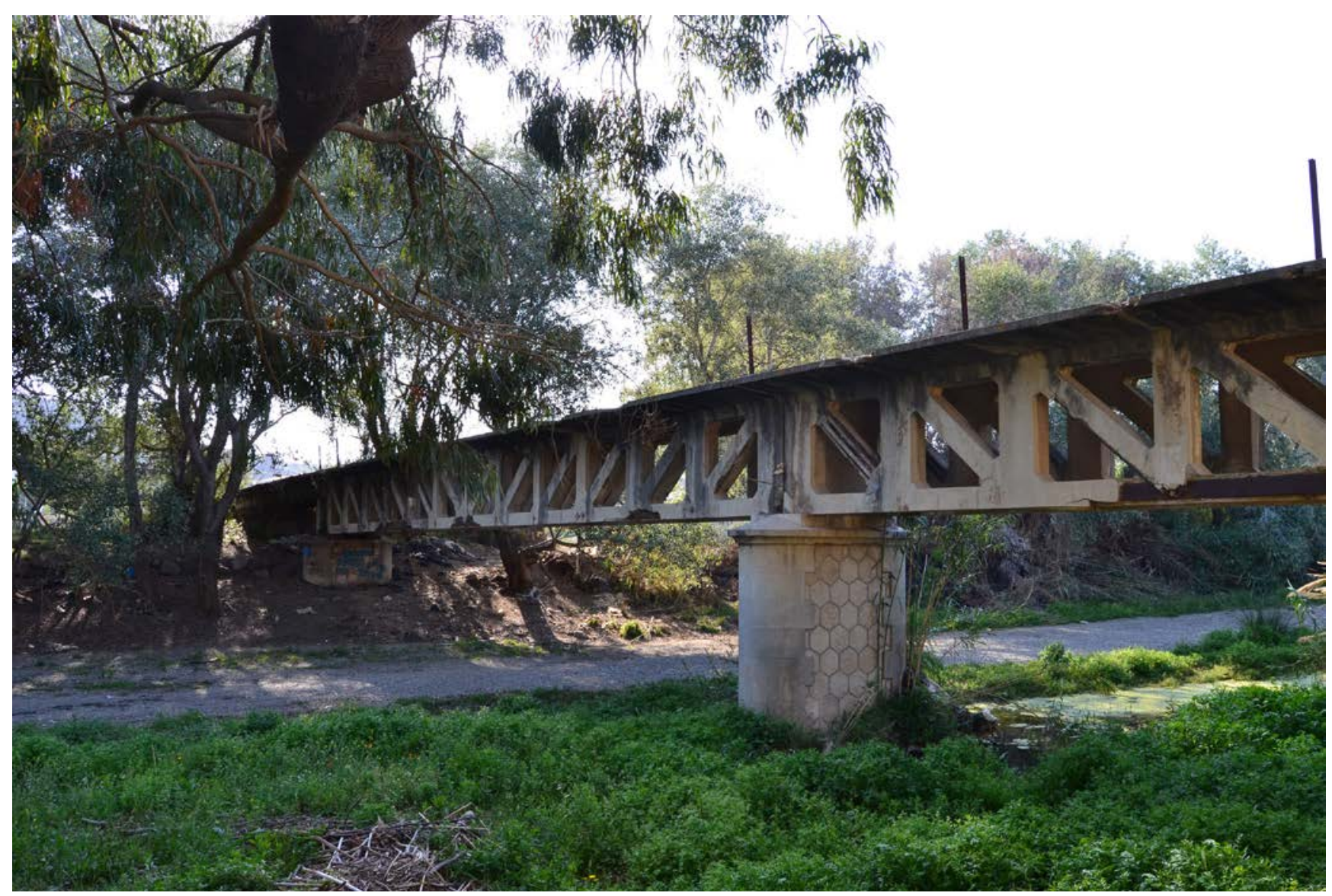

Figura 11. El puente sobre el río Vélez en la actualidad. Se encuentra en avanzado estado de deterioro y en peligro de desaparición. Fotografía del autor.

de la sustitución de la ya anacrónica ferralla de pletinas por barras convencionales, el cambio más trascendente fue la utilización de una configuración Howe, en la que se invertía el esquema de trabajo de montantes y diagonales. Consciente de que una de las grandes debilidades del modelo era su complicada ejecución, Zafra decidió este cambio en aras de aliviar la realización de esta tarea (14).
Avalados ya por su normalización reglamentaria, los puentes de vigas trianguladas tuvieron cierto protagonismo en España hasta la Guerra Civil. Se construyeron en número relevante, con resultados satisfactorios. Después, debido principalmente a las dificultades de ejecución, serían apartados definitivamente del repertorio creativo de los ingenieros españoles.

\section{REFERENCIAS}

(1) Aguiló, M. (2008). Forma y tipo en el arte de construir puentes. Madrid: Abada.

(2) Kersten, C. (1909). Brücken in Eisenbeton. Berlin: Verlag von Wilhelm Ersnt \& Sohn.

(3) Sáenz Ridruejo, F. (1993). Los ingenieros de Caminos. Madrid: Colegio de ingenieros de Caminos, CC y PP.

(4) Navarro, J.R. (2001). El puente moderno en España. Madrid: Fundación Juanelo Turriano.

(5) Burgos, M. (1976). Los ferrocarriles suburbanos de Málaga, S.A. Jábega, 13, 6-11.

(6) Werner, L (1906). Compañía de los Ferrocarriles Suburbanos de Málaga. Proyecto general de Obras de Fábrica y Puentes. Archivo General de la Administración, (04)087, 25/8094.

(7) De Zafra, J.M. (1907). Compañía de los Ferrocarriles Suburbanos de Málaga. Proyecto de puente de hormigón armado sobre el río Vélez. Archivo General de la Administración, (04)087, 25/8095.

(8) Christophe, P.(1902). Le béton armé et ses applications. Paris: Librairie Polytechnique Ch. Béranger.

(9) Dumas, A. (1900). Travaux publics. Ligne de Courcelles au Champ-de-Mars, à Paris. Travaux de la partie comprise entre Courcelles et Passy. Le Génie Civil, 37(943), 1-12.

(10) De Zafra, J.M. (1911). Construcciones de hormigón armado. Madrid: Imp. y Enc. de V. Tordesillas.

(11) De Zafra,J. M. (1910). Puentes de hormigón armado para ferrocarril. Revista de Obras Públicas, 58(1883), 1-6.

(12) Mendizábal, A. (1943). Explotación de ferrocarriles por el Estado. Memoria relativa al periodo $1^{\circ}$ julio 1941-3o diciembre 1942. Madrid: Artes Gráficas Faure.

(13) Ribera, J.E. (1932). Puentes de Fábrica y de Hormigón Armado, tomo IV. Madrid: Suc. Rivadeneyra.

(14) De Zafra, J.M. (1921). Modelos de Puentes de Hormigón Armado para Carreteras. Archivo General del Ministerio de Fomento. 